\begin{tabular}{|c|c|c|c|}
\hline Eiszeitalter und Gegenwart & 53 & $\begin{array}{c}26-38 \\
7 \mathrm{Abb} ., 1 \mathrm{Tab}\end{array}$ & Hannover 2003 \\
\hline
\end{tabular}

\title{
Magnetische Aspekte zum Rodderberg-Vulkan (Bonn)
}

\author{
Rudolf Pucher ${ }^{*}$
}

keywords: Quaternary volcanism, Rhine valley, magnetic surveying, rock magnetism, phreatomagmatism

Kurzfassung: Der Rodderberg bei Bonn ist der nördlichste Teil des Osteifel-Vulkanfeldes aus dem Pleistozän.

Seine mehrphasige Entstehungsgeschichte ist verzahnt mit den fluviatilen Sedimenten des Rheins und den äolischen Sedimenten aus den Eiszeiten. Die dort aufgeschlossenen Gesteine Basalt, Löß und vulkanthermisch beeinflusster Löß erlauben den Vergleich von Magnetisierungen von Materialien etwa gleichen Alters, aber unterschiedlicher Zusammensetzung und Geschichte.

Die von der lokalen Erdmagnetfeld-Richtung deutlich abweichende flache Magnetisierungsrichtung der Basaltproben ist durch Blitzschlag verursacht worden und stellte sich als Beispiel von Blitzschlag-Magnetisierung heraus. Aus der Verteilung der Deklination der Magnetisierung konnte die Einschlagstelle der Blitze in etwa $5 \mathrm{~m}$ vom Probenprofil ermittelt werden.

Eine aeromagnetische Vermessung des Rodderberges hat neue Hinweise zu seiner Struktur ergeben:

Die Anomalie erstreckt sich von NNW nach SSE, der magnetisch wirksame Teil der Vulkanstruktur beschränkt sich auf den Bereich innerhalb des topographisch erkennbaren Kraters. Der umlaufende Schlacke-Aschewall ist magnetisch nicht erkennbar. Unterschiede der Anomalieform aus Messungen am

\footnotetext{
* Anschrift des Verfassers: PD Dr. Rudolf PuCHER Institut für Geowissenschaftliche Gemeinschaftsaufgaben (GGA), Hannover

Email: r.pucher@gga-hannover.de
}

Boden und in $35 \mathrm{~m}$ Höhe (Aeromagnetik) legen den Schluss nahe, dass die magnetisch wirksame Kraterstruktur einen flachgründigen Körper aus pyroklastischem Material anzeigt. Mit einigem Abstand darunter ist weiterer Basalt zu erwarten.

Mit dem Rodderberg scheint uns die Natur einen Vulkan zu liefern, der am Beginn von phreatomagmatischen Erscheinungen geprägt war, dessen zweite Hälfte seiner lang währenden Genese aber die eines mehr klassischen Vulkans war.

\section{[Magnetic Aspects of the Rodderberg Volcano (Bonn)]}

Abstract: The Rodderberg volcano is the most northerly part of the eastern Eifel volcanic field of Pleistocene age. Its complex genesis is connected with the fluviatile Rhine river sediments and with eolian glacial sediments. The neighbouring rocks of basalt, loess and thermal-influenced loess led to a magnetization comparison of samples of roughly the same age but of different composition and history.

The magnetization direction of the basaltic samples is almost horizontal and therefore deviates from the earth's magnetic field. Its origin turned out to be caused by being struck by lightning. It was possible to determine the place of lightning to be about $5 \mathrm{~m}$ south of the sample's location.

An aeromagnetic survey of the Rodderberg has revealed some new information regarding its structure: The magnetic anomaly shows a striking in NNWSSE direction, the magnetically sensitive part of the volcano is restricted to the area inside the topographic crater. The ring wall of scoria and ashes is not detectable magnetically. Differences in anomaly shape of magnetic measurements at surface and at $35 \mathrm{~m}$ height (aeromagnetics) may identify the shallow-seated ma- 
gnetically sensitive part of the crater as by phreatomagmatism caused pyroclastic part of a maar. After some interruption more basaltic material is expected. It appears that the Rodderberg volcano is an example of nature where at the beginning the genesis was ruled by phreatomagmatism but its activity has ended up with a basaltic body due to normal magma crystallization.

\section{Einleitung}

Der Rodderberg, zwischen den Ortschaften Rolandswerth und Niederbachem südlich von Bonn gelegen (s. Übersichtsteil in Abb. 1), ist der nördlichste Teil des Osteifel-Vulkanfeldes aus dem Pleistozän (VAn DEN BOgaArd et al. 1989). Heutigen Zugang zum Rodderberg bilden lediglich einige als Naturdenkmal ausgewiesene Klippen innerhalb der nördlichen Schlackenfelder. Der Rodderberg selbst mit 195 m NN liegt innerhalb des südlichen Schlackenwalls. Da das vulkanische Gestein den Löß durchschlagen hat, ist der Rodderberg jünger als dieser Löß.

Durch Vergleich der Magnetisierungsrichtung der im vulkanischen Gestein als thermoremanente Magnetisierung (TRM, Abkürzungen s. Glossar) beim Abkïblen "eingefrorenen" Erdfeldrichtung mit der Sedimentationsremanenz (DRM) an Löß-Sediment lässt sich der Grad der Richtungsübereinstimmung ermitteln; denn die Magnetisierung in Lößmaterial wird überwiegend als Ergebnis der Ablagerung feinster magnetischer Partikel in Erdfeldrichtung verstanden.

Darüber hinaus ist zu erwarten, dass das LößMaterial im thermischen Kontakt zur heißen Lava - erkennbar an Verfestigung, Frittung und Verfärbung - eine TRM oder CRM erwirbt, die die Richtung des Erdmagnetfeldes besser widerspiegeln wird als die Sedimentationsremanenz. Der Rodderberg bietet also die Möglichkeit, Thermoremanenz, Chemische Remanenz und Sedimentationsremanenz quartären Alters vergleichen zu können.
Wanderer können darüber hinaus die irritierende Beobachtung machen, dass beim Passieren der Basaltklippe die Nadel des Kompasses eine volle Umdrehung vollführt. Das heißt, dass das dorrige Gestein einen so starken Magneten darstellt, dass dessen Magnetfeld stärker als das dortige Erdmagnetfeld ist.

\section{Geologisch-tektonische Einführung}

Richter (1942) hat alle damals möglichen Beobachtungen in einer geologischen Exkursionskarte zusammengefasst und bemerkenswerte Schlussfolgerungen gezogen. Da damals Schlacken und Aschen für den Wegebau abgebaut wurden, nahm das Wissen um den Rodderberg durch zahlreiche Exkursionen, hauptsächlich des Geologischen Institutes Bonn, stetig zu. Die wichtigsten Elemente seien hier zusammengestellt:

- Die Entstehung des Rodderberges ist nicht ein einmaliges Ereignis, sondern das Ergebnis einer Serie von Eruptionen, deren Ausbruchspunkte nicht konstant blieben. Zeitlich liegen sie zwischen dem Ende der ,älteren Hauptterrasse" (fluviatile Sedimente des Rheins) und den „jüngeren Hauptterrassen“. Die älteren Tuffe liegen konkordant zu und gleichartig eingeregelt wie die Rheinsediment-Konglomerate.

- Der weit verbreitete Löß ist unterschiedlichen Alters: Lavagänge (im Westen und Nordwesten) haben den älteren Löß und auch die unteren Schlackenhorizonte durchschlagen. Andere Löß-Horizonte sind eindeutig jünger als alle vulkanischen Spuren.

- Aus dem Mißverhältnis von der Größe des Kraterdurchmessers (ca. $800 \mathrm{~m}$ ) und der geringen nachweisbaren Schlackenmenge wurde auf eine Maar-Struktur geschlossen. Auswürflinge, die bis $\mathrm{zu} 0,5 \mathrm{~m}$ mächtige gefrittete Devonbrocken enthalten, sprechen ebenfalls dafür. 
Mit den Fortschritten der Löß-Stratigraphie hin zu deutlich unterschiedlich alten Löß-Horizonten und der Glazialforschung nahm das vermutete Eruptionsalter des Rodderberges zu; noch FreChen (1976) assoziiert ein Alter von 30.000 a der Eruption, abgeleitet aus dem in einer kraterartigen Vertiefung von ca. $40 \mathrm{~m}$ anstehenden Löß.

Das Bohrprofil einer neuen Bohrung ins Zentrum des Tephraringes - der Forschungsbohrung Rodderberg 1/2000 des Geologischen Dienstes Nordrhein-Westfalen - lässt unter Verwendung aller Literaturdaten drei Eruptionsphasen annehmen, die durch lange Hiaten voneinander getrennt sind (BLANCHARD \& Zöller 2002). Für die jüngste Phase wird ein Base Surge mit nachfolgenden Ausfällungen von Tephra mit verbackenen DevonschieferPartikeln gefunden, ein weiteres Anzeichen für Phreatomagmatismus. BLANCHARD \& ZÖLLER (2002) haben mit Hilfe von Schiefer-Xenolithen aus basaltischem Lavamaterial Thermolumineszenz-Bestimmungen (TL) durchgeführt, deren vorläufige Ergebnisse ein Eruptionsalter >> 200.000 a ergeben.

\section{Magnetfeld-Anomalien am Rodderberg}

Das Gebiet des Rodderberges wurde im Jahr 1978 durch die Bundesanstalt für Geowissenschaften und Rohstoffe (BGR), Hannover, im Rahmen von Erprobungsarbeiten aeromagnetisch vermessen (SENGPIEL 1981). Deren Ergebnis soll an dieser Stelle vorgestellt und diskutiert werden. In Abb. 1 ist der Isolinienplan der Intensität des erdmagnetischen Feldes mit der Lage der Flugprofile und den ungefähren Koordinaten im Gauß-Krüger-System, in ein Luftbild einkopiert, dargestellt. Die Vermessung erfolgte mit Hilfe eines Hubschraubers durch ein Protonenpräzessionsmagnetometer.

Der Sensor befand sich in konstantem Abstand von $35 \mathrm{~m}$ über Gelände, der Abstand der Messpunkte beträgt etwa $30 \mathrm{~m}$. Dargestellt sind die Isolinien der Totalintensität F; seit 1978 ist F um etwa $550 \mathrm{nT}$ gestiegen.

Das Anomaliebild des Rodderberges (s. Abb. 1), in Teilen schon durch Vogeisang (1958) erfasst, wird geprägt durch ein SSE-NNWverlaufendes magnetisches Maximum von etwa $+800 \mathrm{nT}$ (das Null-Niveau wird aus dem Isolinienbild bei $47.750 \mathrm{nT}$ festgelegt) mit einigen lokalen Minima und Maxima im Norden, Folge der dortigen Tuff-Verbreitung und Basaltintrusionen. Der Isolinienabstand beim Hauptmaximum ist nahezu konstant, im Norden geringfügig vergrößert. Der genaue Vergleich von topgraphischer Karte und der Darstellung von Abb. 1 ergibt, dass die Erstreckung des magnetischen Vulkanitkörpers sich auf das Gebiet innerhalb der dem Isolienverlauf folgenden Straße beschränkt. Der Schlackenund Aschewall ist magnetisch nicht erkennbar. Der Rodderberg selbst liegt außerhalb der Hauptanomalie und ist an der lokalen Anomalie mit dem Maximum-Wert + 47.915 nT (dessen Ursache können die dortigen Gebäude sein) nur erkennbar. Das trifft ebenfalls für das Gut Rodderbergshof im Süden (erkennbar an der Ausbuchtung der Isolinie $47.750 \mathrm{nT}$ ) zu. Lediglich die lokalen Maxima $+47.867 \mathrm{nT}$ und $47.704 \mathrm{nT}$ im Norden stören das klassische Anomaliebild eines ebenmäßigen magnetischen Quellkörpers mit einer dem heutigen Erdmagnetfeld ähnlichen Neigung der Magnetisierung mit $\mathrm{I}=66^{\circ}$ (Maximum mit einem im Norden vorgelagerten, kappenähnlichen Minimum).

Da die Arbeiten zur aeromagnetischen Vermessung auch Magnetfeldmessungen am Boden einschlossen, eröffnet sich die Möglichkeit, über einen Vergleich der Messwerte in zwei unterschiedlichen Niveaus magnetische Hinweise auf die Kraterstruktur zu erhalten. Dafür wurden die Messwerte beider Messniveaus in einem NNW-SSE-Profil (Lage ist in Abb. 1 eingetragen) entnommen. Der Profilvergleich ist in Abb. 2 dargestellt. Wichtigster Unterschied der 


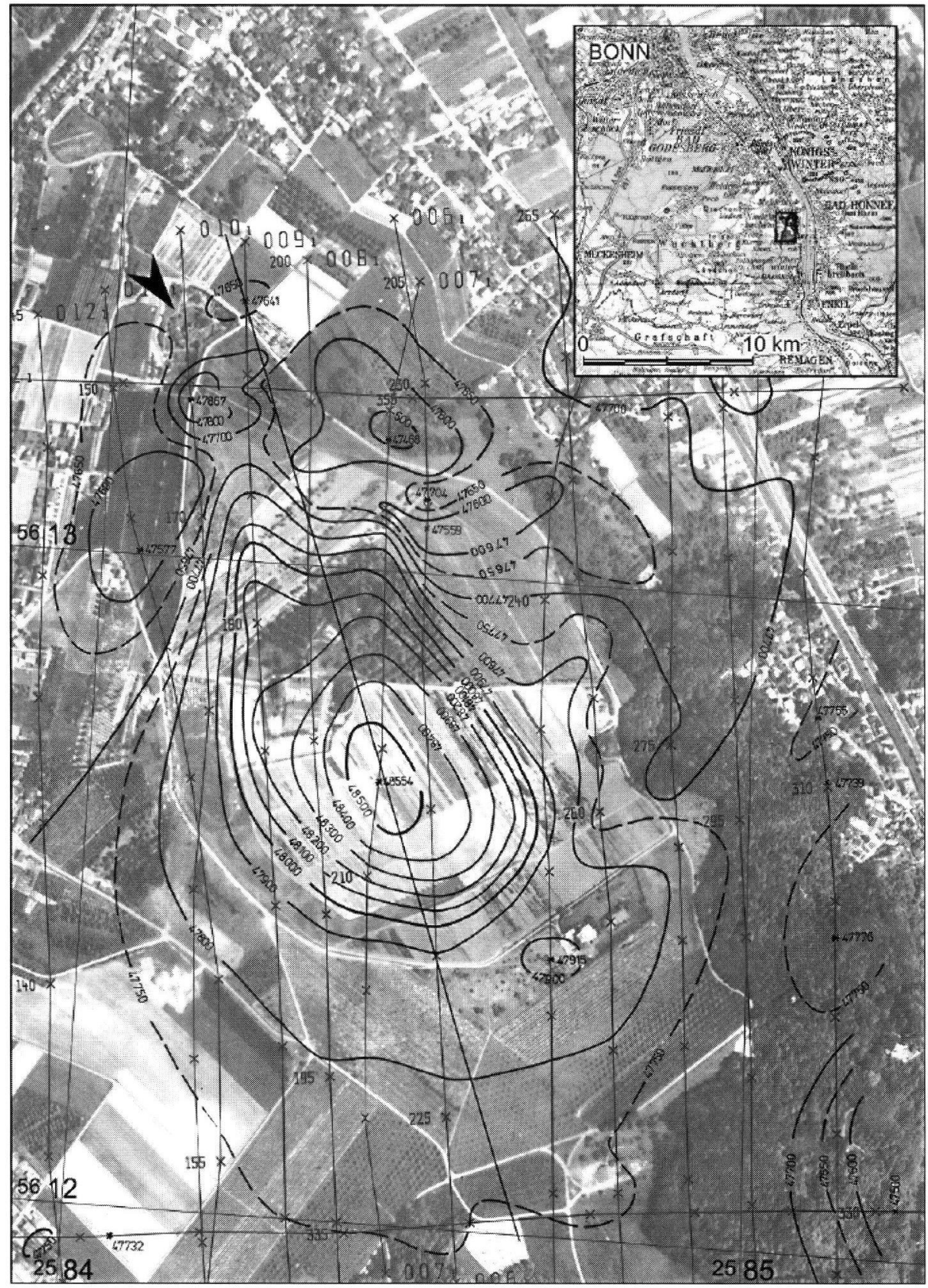

Abb. 1: Aeromagnetische Vermessung des Rodderberges bei Mehlem (Bonn) nach SEngPiel (1981) in 35 $m$ über Gelände. In das Luftbild sind eingetragen das Flugprofil-Netz und die Isolinien der erdmagnetischen Totalintensität F für August/1978. Linie SSE-NNW: Profil s. Abb. 2, Pfeil: Lage der BlitzschlagMagnetisierung, Gauß-Krüger Koordinaten.

Fig. 1: Aeromagnetic survey of the Rodderberg near Mehlem (Bonn) after SEnGPIEL (1981) with a constant altitude of $35 \mathrm{~m}$. Onto an aerial photograph the flight lines and the isolines of the earth's magnetic intensity F for August 1978 are drawn. Line SSE-NNW: profile s. Fig. 2, arrow: lightning magnetization, GaussKrüger coordinates. 
Profile in unterschiedlichem Niveau ist im Bodenprofil eine „Delle“ nördlich des Maximums, die in dem Profil $35 \mathrm{~m}$ über Gelände nicht mehr zu erkennen ist. Die Differenzkurve (Abb. 2 unten) gibt den Feldunterschied quantitativ wieder. Sie beschreibt die Feldverteilung eines flachgründigen, nur Dekameter mächtigen plattenförmigen magnetischen Quellkörpers - im Süden ein Maximum und im Norden ein Minimum. Die Kraterstruktur erreicht nach geoelektrischen Messungen eine Tiefe von mehr als $50 \mathrm{~m}$ (Thiemer \& Tezkan 2002).

Eine Ringstruktur magnetisch wirksamen Materials kann ausgeschlossen werden, weil in

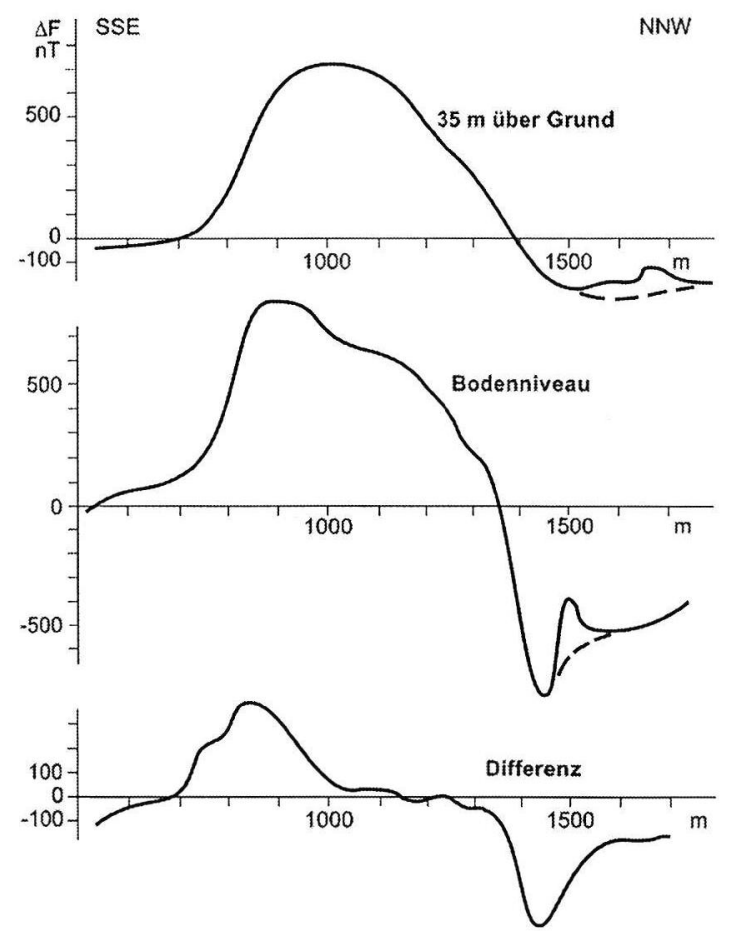

Abb. 2: Profilvergleich der magnetischen Anomalie in SSE-NNW-Richtung ( $s$. Abb. 1) in Bodenniveau (Mitte) und $35 \mathrm{~m}$ über Grund (oben). Die Differenzkurve (unten) deutet auf einen flachgründigen Quellkörper hin.

Fig. 2: Magnetic profiles SSE-NNW (s. Fig. 1) $35 \mathrm{~m}$ above the ground (top) and at surface (middle). The differential curve (below) indicates a shallow magnetic body source. einem solchen Fall am Nordrand der Vulkanstruktur ein die Wallstruktur nachzeichnendes Maximum erkennbar sein müsste. Zweifelhaft ist die Zuordnung des stark ausgebildeten Minimums im Norden. Falls das Minimum Begleitanomalie zum Hauptmaximum ist, ist ein südliches Einfallen des basaltischen Hauptkörpers möglich. Der Aufbau der magnetisch wirksamen vulkanischen Struktur stellt sich so dar, dass innerhalb des Kraters ein nur Dekameter mächtiger flachgründiger plattenförmiger Quellkörper nach einer unmagnetischen Einschaltung vermutlich von einem basaltischen Gesteinskörper unterlagert wird.

\section{Probennahme für die Magnetisierungstests}

Das kurze Profil der Basaltproben zur Untersuchung der Blitzschlag-Magnetisierung liegt am Nordrand der Klippe in Richtung $60^{\circ} \mathrm{W}$ (R: $2584,053 \mathrm{~km}, \mathrm{H}: 5613,295 \mathrm{~km}$, Pfeil in Abb. 1). Es wurden fünf Kerne im Meterabstand erbohrt. Auflage der Denkmalschutzbehörde war, die Löcher mit den oberen Kernresten wieder zu verschließen. Die magnetische Orientierung musste wegen der Kompass-Störungen am Probenentnahmepunkt mit Peilungen in etwa 20 $m$ Abstand erfolgen.

Das Löß-Profil konnte in zehn Probenpunkten den systematischen Wechsel vom Tuffkontakt bis zum ungestörten Löß in $2000 \mathrm{~m}$ Entfernung (Tab. 1) erfassen. Die Entnahme erfolgte z.T. mit einem Probenstechrohr, teils durch große Probenkörper, die im Labor zu Kleinproben zerteilt wurden.

\section{Gesteinsmagnetik}

Die Laboruntersuchungen umfassten die vektorielle Ermittlung (Intensität und Richtung) der natürlichen remanenten Magnetisierung (NRM; Abkürzungen s. Glossar) und ihrer Stabilität 
Tabelle 1: Gesteinsmagnetische Befunde

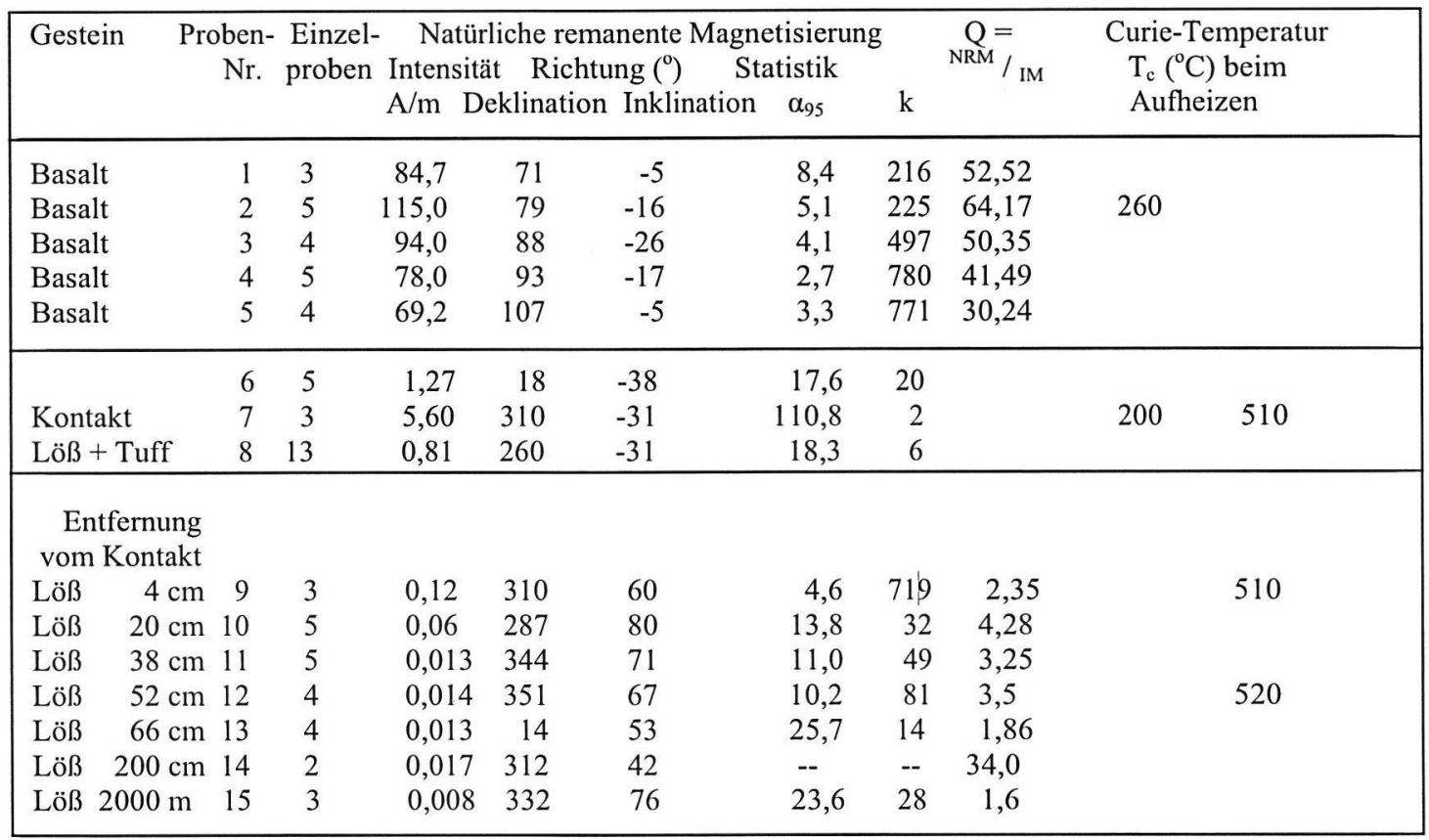

nach progressiver Entmagnetisierung mit magnetischen Wechselfeldern und nach thermischer Entmagnetisierung. Aus der ebenfalls bestimmten induzierten Magnetisierung (IM) kann im Verhältnis zur NRM der Königsberger Faktor $\mathrm{Q}={ }^{\mathrm{NRM}} / \mathrm{IM}$ berechnet werden. In Tab. 1 sind die gesteinsmagnetischen Befunde zusammengestellt. Zur Betrachtung der NRM-Richtung sind außer den Mittelwerten der Deklination und Inklination statistische Angaben zur Streuung innerhalb eines Handstückes gegeben: $\alpha_{95}$ beschreibt den Radius des Konfidenz-Kegels, innerhalb dessen die Einzelwerte mit einer Wahrscheinlichkeit von $95 \%$ liegen, und $\mathrm{k}$ ist ein Präzisionsparameter.

An Pilotproben wurde in der thermomagnetischen Analyse durch die Messung der Sättigungsmagnetisierung beim Aufheizen bis $680^{\circ} \mathrm{C}$ und Abkühlen die thermische Stabilität untersucht sowie die Curie-Temperatur $\mathrm{T}_{\mathrm{c}}$, oberhalb der sich ferro- und ferrimagnetische Stoffe paramagnetisch verhalten, ermittelt (Messbeispiele s. Abb. 3). Zu Vergleichszwe- cken wurde an einer Probe eine künstliche Thermoremanenz erzeugt, indem die Probe nach Erhitzen bis $680^{\circ} \mathrm{C}$ im Erdmagnetfeld abgekühlt wurde.

Löß: Löß ist ein äolisches Sediment als Ergebnis von Wind, Frost, Gesteinsabrieb durch Eismechanik (Eiszeiten), Verwitterung und biologischen Prozessen und besteht hauptsächlich aus Quarz- und Feldspat-Partikeln (für eine Übersicht s. Heller \& Evans 1995). Eingeschaltete fossile Bodenhorizonte, in denen der Löß umgelagert und durch Verwitterung z.T. zu Tonmineralen umgewandelt ist, zeigen Zwischen-Warmzeiten (Interstadiale) an. Die Schwermineralfraktionen enthalten als magnetische Minerale Magnetit, Maghemit, Hämatit und Goethit, für deren Ursprung auch Magnetitkörner (mit Korndurchmessern $\ll 100$ $\mu \mathrm{m})$, auch als Wirkung von magnetotaktischen Bodenbakterien (BlaKemore 1975, FassBinDER et al. 1990) angenommen werden. Dabei liefern die Richtungen der remanenten Mag- 

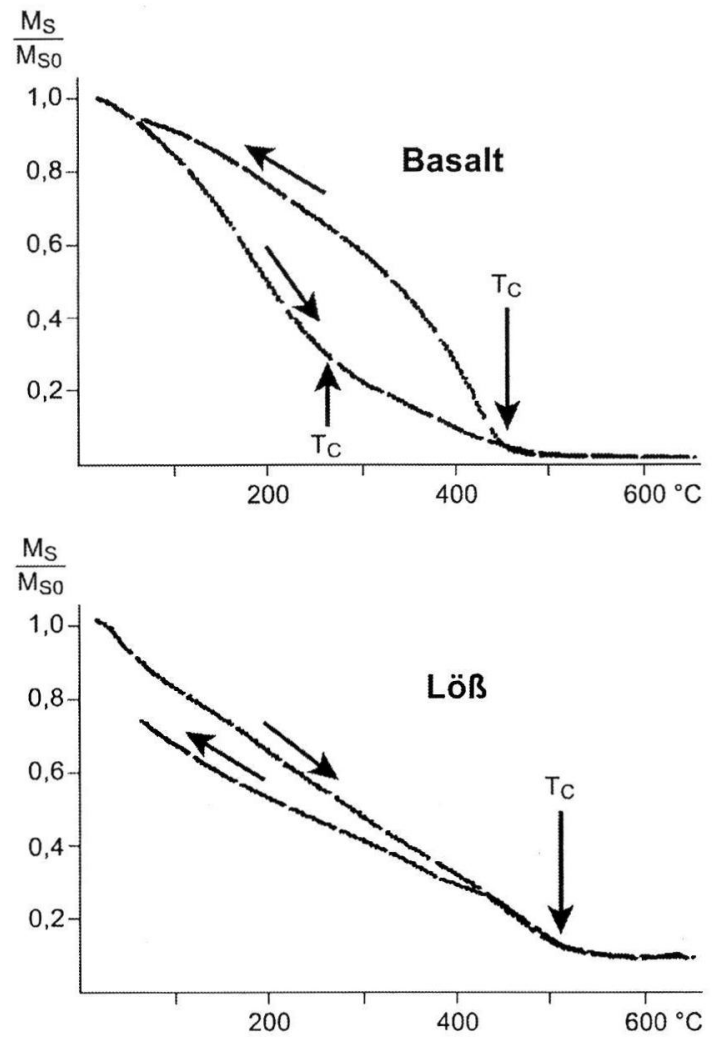

Abb. 3: Thermomagnetische Analysen einer Basalt(oben) und einer Lößprobe (unten): In dem starken magnetischen Gleichfeld von $400 \mathrm{mT}$, durch das fast alle magnetischen Bereiche der Probe ,ausgerichtet" werden (Sättigung), wird das magnetische Moment Ms als Funktion der Temperatur beim Aufheizen und Abkühlen gemessen und auf seinen Anfangswert Mso normiert aufgetragen. Aus dem Verlauf der Aufheizkurve wird der Wert der Curie-Temperatur $T_{c}$ bestimmt; aus dem Vergleich der Aufheiz- und Abkühlkurve wird die thermische Stabilität geprüft.

Fig. 3: Thermomagnetic analyses of a sample of basalt (upper) and of loess (lower): In a strong DC magnetic field $(400 \mathrm{mT})$ in which most magnetic domains are aligned (,saturation") the magnetic moment Ms is measured as a function of temperature during the sample's heating and cooling. This is normalized to its starting value Mso and plotted. From the shape of the heating curve the value of the Curie temperature $T_{c}$ is determined. The comparison of the heating and cooling curve thermal stability is tested. netisierung die konservierten erdmagnetischen Polaritätswechsel der letzten 2-3 Ma. Die exakte Konservierung der Erdmagnetfeld-Richtung ist nur bei ausnahmsweise günstigen Ablagerungsbedingungen erfolgt. Die magnetische Suszeptibilität als Maß des Gehalts an magnetisierbarem Material liefert andererseits paläoklimatische Informationen über Warm- und Kaltzeiten.

Löß ungestört: Die Richtungen der remanenten Magnetisierung (Tab. 1 unten), insbesondere der Inklination, stimmen unter Zuhilfenahme der Konfidenzwinkel-Werte gut mit der magnetischen Erdfeldneigung Tertiär bis heute $\left(\mathrm{I}=63^{\circ}-67^{\circ}\right.$; lokale heutige Erdfeldrichtung Sternsymbol in Abb. 5) überein. Eine starke viskose Magnetisierung VRM ist nicht feststellbar. Die remanente Magnetisierung NRM ist stärker als die durch das heutige Erdmagnetfeld induzierte $(\mathrm{Q}>1)$. Die gemessene Curietemperatur $\mathrm{T}_{\mathrm{c}}=510^{\circ} \mathrm{C}$ lässt Magnetit als Träger der Magnetisierung erkennen. Der hier aufgeschlossene Löß erweist sich mit seiner Magnetisierung als geeignet für magnetostratigraphische Studien.

\section{Löß, im Kontakt zum Basalt thermisch} gefrittet (Tab. 1 Mitte): Der nur ca. $3 \mathrm{~cm}$ starke Kontaktsaum von Löß und Basalt ist ein Gemenge von Löß- und Schlacketeilchen. Die Werte der remanenten Magnetisierung streuen sowohl in ihrer Intensität als auch Richtung beträchtlich. Die verziegelten Lößanteile tragen vergleichsweise wenig zur Gesamt-Magnetisierung bei. Auffallend sind die Magnetisierungsrichtungen. Die doch recht gleichartigen negativen Inklinationswerte sind nicht korrelierbar mit der Erdfeldrichtung, weder während der vulkanischen Überprägung noch durch die Lagerung im Erdmagnetfeld seitdem. Nach progressiver Abmagnetisierung mit magnetischen Wechselfeldern nimmt die Intensität schnell ab, und die Richtung der jeweiligen Restremanenz ändert sich. Es wird festgestellt, dass in diesem Kontaktbereich die Richtung des Erdmagnetfeldes nicht ungestört konserviert worden ist. 
Basalt: Die magnetische Suszeptibilität weicht mit dem Mittelwert von $49 \cdot 10^{-3}$ SI (Tab. 1 oben) nicht von den für Basalte üblichen Werten (Reynolds 1998) ab. Das ist aber für die Intensität und die Richtung der remanenten Magnetisierung der Fall: Mit bis zu $115 \mathrm{~A} / \mathrm{m}$ sind diese Proben mehr als zehnmal so stark magnetisiert wie normale Basalte. Die Richtungsmittelwerte der NRM - ihr Konfidenzkegel ist erstaunlich klein und der Güte-Parameter außerordentlich groß (FISHER 1953) - weichen erheblich von derjenigen $\mathrm{ab}$, die man für TRMWerte erwarten sollte (Sternsymbol in Abb. 5). Die grob nach $\mathrm{E}$ und mit $20^{\circ}$ nach oben weisenden NRM-Werte $\left(\mathrm{I} \sim-20^{\circ}\right)$ verlieren bei progressiver Entmagnetisierung schnell an Intensität, nach Entmagnetisierung mit 20 nT beträgt die Restremanenz weniger als $5 \%$ des Anfangswertes. Dabei wird die Richtung der Restremanenzen zunehmend steiler (Abb. 4 unten). Die gemessene Curie-Temperatur von 260 ${ }^{\circ} \mathrm{C}$ lässt einen Titanomagnetit mit beträchtlichen Ti-Gehalt erkennen (O'REILLy 1984).

\section{Blitzschlag-Magnetisierung}

Magnetostatische Felder, die in ihrer Stärke das Erdmagnetfeld (bei uns etwa $48.000 \mathrm{nT}$, s. Abb. 1) übertreffen, werden nur von seltenen Magnetit-Lagerstätten berichtet, z.B. Kiruna/ Schweden oder Tschogart/Iran (HAHN \& Bosum 1986). Eine andere Möglichkeit extrem hoher Magnetfelder ist Blitzschlag-Magnetisierung. Einer der Hinweise darauf ist eine nahezu horizontale Magnetisierung, die bei einer vertikalen Blitzbahn längs der orthogonal dazu verlaufenden ringförmigen magnetischen Feldlinien entsteht. Diese Isothermale Remanenz (IRM), also ohne erhöhte Temperatur erworbene Remanenz und in unserem Fall identisch mit der heutigen Feldrichtung, ist, wie alle anderen Remanenzen, in ihrer Intensität proportional zur Stärke des angelegten äußeren Magnetfeldes. Das bestätigt auch ein Experiment, in dem in einer der Proben durch Erhitzung auf $680^{\circ} \mathrm{C}$, also deutlich über ihre Curie-Temperatur, und anschließende Abkühlung im Erdmagnetfeld eine künstliche Thermoremanenz TRM erzeugt wurde. Sie erreicht im Messbefund bei gleich bleibender Suszeptibilität nur etwa 10 Prozent der NRM. Aus Abb. 6 wird deutlich, dass die im Labor

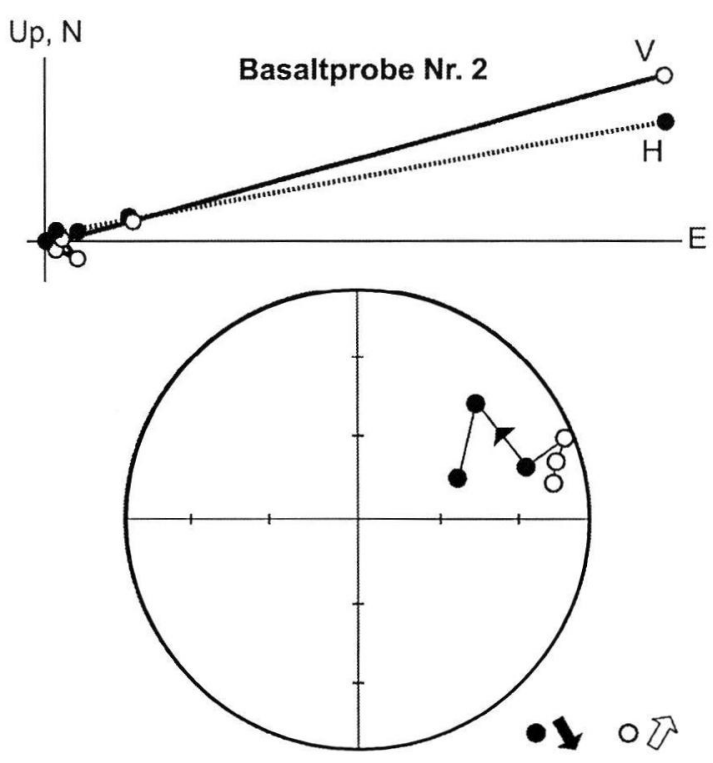

Abb. 4: Veränderung der remanenten Magnetisierung der Basaltprobe Nr. 2 (vgl. Tab. 1) bei progressiver Entmagnetisierung. Oben: Die Darstellung im Zijderveld-Diagramm (Zijperveld 1967) stellt die Projektionen der Werte in die senkrechte NSEbene (offene Symbole) und die horizontale Ebene (geschlossene Symbole) dar. Unten: Darstellung in Polarprojektion ist eine Projektion der Einheitskugel mit den Durchstoßpunkten der Remanenz-Vektoren nach jedem Abmagnetisierungsschritt.

Fig. 4: The change of the remanent magnetization of basalt sample no. 2 (s. Tab. 1) during increasing AF demagnetization. Above: The Zijderveld-plot (Zijderveld 1967) combines the graphs of the vertical NS-plane (open symbols) and the horizontal plane (solid symbols). Below: The stereograph shows the horizontal projection of the unite sphere with the vector points of each partial vector. 


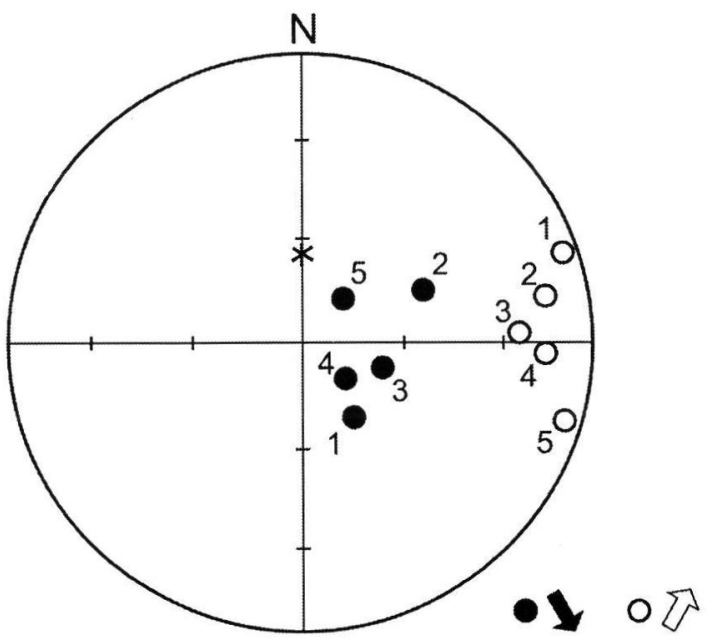

Abb. 5: Polarprojektion der durch Blitzschlag magnetisierten Basaltproben (s. Tab. 1). Offene Symbole (obere Halbkugel): Flach nach oben und Westen weisende NRM-Werte mit systematischem Richtungswechsel der Proben im Profil (vgl. Abb. 7). Volle Symbole (untere Halbkugel): Mittelsteil nach unten weisende Remanenzrichtungen nach Entmagnetisierung mit 150/200 mT.

Fig. 5: A stereograph of the magnetized basalt samples which were struck by lightning (s. Tab. 1). Open symbols (upper hemisphere): Almost horizontal NRM values with systematic directional shift within the profile (s. Fig. 7). Solid symbols (lower hemisphere): Moderately steep downwards remanence directions after demagnetization with $150 / 200 \mathrm{mT}$.

erzeugte TRM gegenüber Wechselfeld-Abmagnetisierung stabiler als die anfangs gemessene NRM ist; Nach einer Abmagnetisierung mit 20 nT beträgt der Restwert der TRM noch $55 \%$, die der NRM nur noch $5 \%$. Aus dem Messbefund wird gefolgert, dass die ursprüngliche TRM durch das starke Blitz-Magnetfeld vollständig überprägt worden ist.

Der Versuch, mit Hilfe des Eintrags der Richtung der Horizontalkomponente der NRM (das sind die Deklinationswerte) in das Probenentnahmeprofil die Lage der Blitzschlagstelle zu konstruieren, gelingt erstaunlich gut. Denn alle Deklinationspfeile in Abb. 7 müssen Tangenten

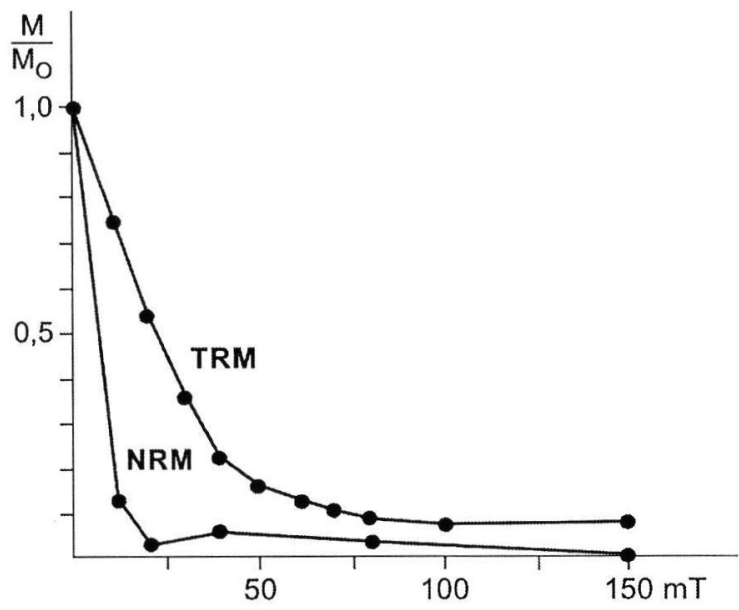

Abb. 6: Stabilitätsvergleich der durch Blitzschlag erzeugten natürlichen remanenten Magnetisierung (NRM) und einer künstlichen Thermoremanenz (TRM) derselben Basaltprobe bei progressiver Wechselfeld-Entmagnetisierung. Die Werte sind auf den Anfangswert normiert.

Fig. 6: A stability comparison of the rema-nent magnetization during increasing AF demagnetization caused by lightning (NRM), and the thermoremanent magnetization (TRM) of the same sample. The values are normalized to the starting value.

der ringförmigen Magnetfeldlinien sein, die denselben Mittelpunkt haben. Es ergibt sich eine Stelle 4,5 m südlich der südlichsten Probe (Abb. 7). Das Entnahmeprofil liegt etwa in NW-SE-Richtung, beginnend mit Probe $1 \mathrm{im}$ NW (s. Tab. 1).

An dieser Stelle seien einige Ergebnisse einer sehr umfangreichen gesteins- und feldmagnetischen Untersuchung und anschließenden rechnerischen Deutung von Blitzeinschlagstellen von Roeser (1963) angeführt. Dabei wurde bis zu einer Tiefe von $2,5 \mathrm{~m}$ in sieben Sohlen an Gesteinsproben die räumliche Verteilung der Magnetisierung ermittelt: Es ergaben sich 


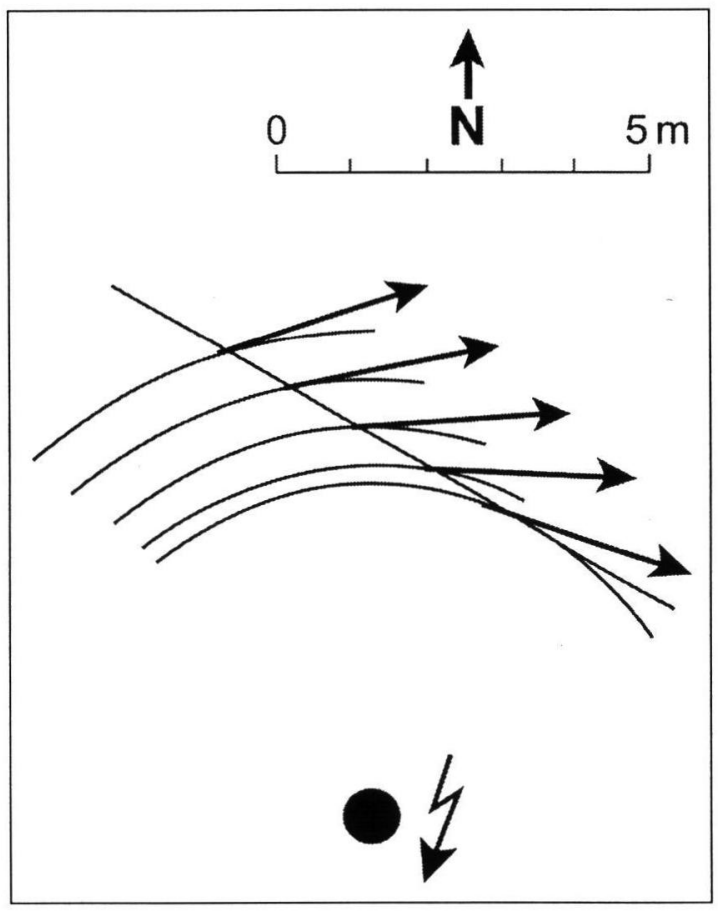

Abb. 7: Ableitung der Blitzschlag-Stelle aus den horizontalen Remanenz-Richtungswerten der NRM in den Probenpunkten als Tangente des ringförmigen Blitz-Magnetfeldes, das durch das vertikale elektrische Feld des Blitzes entsteht. Das Probenprofil beginnt im Nordwesten (offene Symbole 1-5 in Abb. 5).

Fig. 7: Derivation of the place of lightning from the horizontal remanence direction values of the NRM at the places of the collected samples as tangent of the circular magnetic field which has occurred through the vertical electric field of the lightning. The sample-profile starts in northwest (open symbols 1-5 of Fig. 5).

Magnetisierungswerte bis zu $300 \mathrm{~A} / \mathrm{m}$. Auf Grund der Felddaten führen die Rechnungen zu einer etwa senkrechten Blitzbahn mit einer Scheitelstromstärke von ca. $250.000 \mathrm{~A}$, die im Boden auffächert und mit zunehmender Tiefe den Weg größter Leitfähigkeit im Untergrund wählt.

\section{Ergebnisse}

Die aeromagnetische Vermessung der Vulkanstruktur Rodderberg hat neue Hinweise zu seiner Struktur ermöglicht: Der magnetisch wirksame basaltische Körper verläuft von NNW nach SSE. Er schließt nicht den SchlackeAsche-Wall ein, der noch in der Topographie erkennbar ist. Eine merkliche radial nach außen zunehmende Magnetisierungsverteilung wird ausgeschlossen. Die deutlichen Unterschiede der Anomaliestruktur aus dem Vergleich der Aeromagnetik mit Magnetfeldmessungen am Boden lassen einen nur Dekameter mächtigen flachgründigen plattenförmigen Quellkörper innerhalb des Kraters erkennen, mit einer unmagnetischen Einschaltung, die von einem vermutlich basaltischen Gesteinskörper unterlagert wird. Im Nordteil der Anomalie ist das Schlacken-Asche-Feld mit magnetisch wirksamem Material durchsetzt. Eine lokale positive Anomalie von ca. $200 \mathrm{nT}$ im Norden befindet sich ca $70 \mathrm{~m}$ südlich derjenigen Stelle im eingeblendeten Luftphoto, an der die Blitzschlagstelle liegt (Pfeil in Abb. 1).

Die Sicht des Rodderberg-Vulkans als MaarStruktur weckt starkes Interesse in Bezug auf die Maar-Forschung, die in den letzten Jahren intensiv auch im GGA-Institut Hannover betrieben wurde. Dabei war den untersuchten Maar-Strukturen (Baruth (Sachsen): Pucher et al. 2003, im Heft 1/2003 der Z. f. angew. Geol. sind sieben Arbeiten über die Maar-Struktur Baruth erschienen, Messel (Darmstadt): Gabriel et al. 2000, Pucher \& Rolf 2001) gemeinsam, dass ein durch phreatomagmatische Explosionen erzeugter und in der Folgezeit mit „leichtem" Sedimentmaterial verfüllter Kratersee ein rundliches Schwereminimum verursacht, und dass eine damit korrelierte magnetische Anomalie stark magnetisiertes vulkanisches Material unterhalb der Sedimente anzeigt.

Aus den Magnetfeldwerten im Gebiet des Rodderberges und den Differenzwerten aus 35 $\mathrm{m}$ Höhenunterschied ist zu folgern (Abb. 2), 
dass der Hauptteil der magnetischen Anomalie im Zentrum des Kraterbereichs von einem zu erwartenden basaltischen Körper im Schlotbereich erzeugt wird. Der durch die Differenzwerte angezeigte flachgründige Körper könnte aus pyroklastischem Material bestehen. Dabei bleibt offen, ob die magnetische Wirkung ausschließlich induzierter Magnetisierung zuzuschreiben ist, oder ob das vermutete pyroklastische $\mathrm{Ma}$ terial auch eine homogene thermoremanente Magnetisierung aufweist. Das würde beantworten, wie hoch die Ablagerungstemperaturen der Pyroklastika war. Mit dem Rodderberg scheint uns die Natur einen Vulkan zu liefern, der am Beginn von phreatomagmatischen Erscheinungen geprägt war, dessen zweite Hälfte seiner langwährenden Genesegeschichte aber die eines mehr klassischen Vulkans war. Weitere geophysikalische Untersuchungen am Rodderberg erscheinen daher als sehr vielversprechend.

Es bestätigt sich die Eignung von Löß-Material für magnetostratigraphische Studien. Diese Eignung wird in vielen Fällen in unseren Klimabreiten durch komplizierte bodenchemische Reaktionen beeinträchtigt. Daher wird bei Löß in unseren Klimabreiten meist von einer Kombination von primärer Sedimentations- (DRM) und sekundärer chemischer Remanenz (CRM) ausgegangen.

Die Kernproben der Blitzschlag-Magnetisierung ermöglichen eine Rekonstruktion der Blitzstelle: sie liegt ca. $5 \mathrm{~m}$ südlich der Probenpunkte. Die Abweichung der Inklinationswerte von $0^{\circ} \mathrm{kann}$ eine Neigung von $20^{\circ}$ nach $\mathrm{W}$ anzeigen, oder aber Folge der Auffächerung der Strombahn im Boden sein. Es ist anzunehmen, dass infolge der speziellen lokalen Leitfähigkeitsbedingungen sich wiederholt Blitzeinschläge ereignet haben. Im Material des Kontaktbereiches finden sich Titanomagnetite und Magnetite des Löß-Materials, die möglicherweise z.T. erst bei der thermischen Verziegelung entstanden sind. Die flachen Inklinationswerte der Magnetisierung spiegeln in keinem Fall die Richtung des Erdmagnetfeldes in unseren geomagnetischen
Breiten wider. Einzige Erklärungsmöglichkeit ist die Nähe zu einer Blitzschlagstelle zur Zeit der vulkanischen Tätigkeit.

\section{Dank}

Prof. K. Krumsiek hat wegen der KompassAbnormität zu dieser Studie ermuntert. Die Laborarbeiten wurden im Magnetiklabor Grubenhagen des GGA-Instituts unter Leitung von Dipl.-Geophys. K. Fromm durchgeführt, neueste Ergänzungsarbeiten unter der Leitung von Dr. C. Rolf. Die aeromagnetische Befliegung durch die Bundesanstalt für Geowissenschaften und Rohstoffe, Hannover, erfolgte unter der Leitung von Dr. P. Sengpiel, Verm.-Ing. J. Ostwald und Verm.- Ing. H. J. Rehli. Für graphische Arbeiten danke ich Frau J. Herrmann und Herrn Dr. C. Rolf. Das stete Interesse von Dr. M. Frechen und die konstruktiven Anmerkungen der Gutachter haben zu einem verbesserten Manuskript geführt.

\section{Glossar}

TL Thermolumineszenz, wird zur Datierung von thermisch beeinflusstem Material benutzt (Maximalalter $50.000-800.000$ a).

SI Systeme Internationale d'Unites - ein 1954 eingeführtes internationales System von Einheiten, das auf sieben Basiseinheiten beruht.

nT $10^{-9}$ Tesla, Einheit der magnetischen Induktion im SI-System, Magnetfeld.

$\mathrm{A} / \mathrm{m}$ Ampere/Meter - Einheit der magnetischen Feldstärke im SI-System, z.B. bei Entmagnetisierungsprozeduren.

Remanente Magnetisierung (auch „Remanenz") eines Gesteins, ist in Intensität und Richtung (Vektor) abhängig von den Eigenschaften des Erdmagnetfeldes zum Zeitpunkt der Entstehung oder 
Ablagerung und stimmt mit ihm in der Richtung überein; die Informationen bleiben über Jahrmillionen erhalten.

D Deklination der Remanenzrichtung, $0^{\circ}$ $\rightarrow 360^{\circ}$ von $\mathrm{N}$ über $\mathrm{E}$.

I Inklination der Remanenzrichtung gegen die Horizontale; auf der N- Halbkugel $0^{\circ}$ $\rightarrow+90^{\circ}$ (normal magnetisiert), $0^{\circ} \rightarrow-\mathrm{k}$ $90^{\circ}$ (invers magnegtisiert).

NRM Natürliche remanente Magnetisierung, wie man sie nach der Probenentnahme misst; vektorielle Summe aus urspünglicher Remanenz und allen Effekten aus der Lagerung im Erdmagnetfeld seitdem.

TRM Thermoremanente Magnetisierung entsteht durch Abkühlung von Gesteinen im (Erd-)Magnetfeld.

IRM Isothermale remanente Magnetisierung erworben bei Raumtemperatur in einem magnetischen Gleichfeld.

VRM Viskose remanente Magnetisierung, geringe Stabilität bei Entmagnetisierung. Sie ist meist Folge der Lagerung des Gesteins im Erdmagnetfeld.

CRM Chemische remanente Magnetisierung: Durch einen chemischen Prozess kommt es zur Neubildung oder Umwandlung ferrimagnetischer Minerale und somit zur Ausbildung einer CRM in Richtung des vorherrschenden Magnetfeldes.

DRM Sedimentationsremanenz (detrital remanent magnetization) in Sedimenten durch Einregelung magnetisierter Teilchen in Richtung des vorherrschenden Magnetfeldes.

Fisher-Statistik: Standardverfahren in der Paläound Archäomagnetik zur Berechnung der mittleren Remanenzrichtung und deren Fehlerparameter aus Einzelrichtungen (Mittelung von Einheitsvektoren auf der Kugel; Fisher 1953). $\alpha_{95}$ beschreibt den Radius des KonfidenzKegels auf dem Einheitskreis, innerhalb dessen die Einzelwerte mit einer Wahrscheinlichkeit von $95 \%$ liegen. Der Konfidenzwinkel wird bei gleicher Streuung und zunehmender Probenzahl kleiner.

k Präzisionsparameter gibt den Grad der Streuung von Einzelrichtungen wieder (hohe Werte bedeuten geringe Streuung).

Q Königsberger Faktor Q = NRM/IM .

IM Induzierte Magnetisierung: Magnetisierung proportional mit und parallel zum wirkenden Magenfeld F; verschwindet bei $\mathrm{F}=0$.

$\kappa$ magnetische Suszeptibilität (dimensionslose Größe, Skalar) beschreibt die Magnetisierbarkeit eines Minerals und ist in einem Sedimentgestein proportional zum Gehalt magnetisierbarer Minerale. Es gilt: $I M=\kappa \cdot F$.

$\mathrm{T}_{\mathrm{c}}$ Curie-Temperatur, oberhalb der sich ferro- und ferrimagnetische Stoffe paramagnetisch verhalten.

\section{Schriftenverzeichnis}

Blakemore, R. P. (1975): Magnetotactic Bacteria. - Science, 190: 377-379; Washington D. C.

Blanchard, H. \& ZölLER, L. (2002):TL dating of a middle Pleistocene volcano using slate xenoliths. - 10th International Conference on Luminescence and Electron Resonance Dating, Reno (USA), June 2002 (Abstract). Fassbinder, J. W. E., Stanjek, H. \& Vali, H. (1990): Occurrence of magnetic bacteria in soil. - Nature, 343: 161 163; London.

Fisher, R. A. (1953): Dispersion on a sphere. - Proc. Royal Soc. London, 217A: 295-305. FreChEN, J. (1976): Sammlung geologischer 
Führer, Nr. 56. - 3. Aufl.; Berlin (Borntraeger).

Hahn, A. \& Bosum, W. (1986): Geomagnetics, Selected Examples and Case Histories: 2-9; Berlin (Borntraeger).

Helder, F. \& Evans, M. E. (1995): Loess magnetism. - Review Geophysics 33, 2: 211 240; Amer. Geophys. Union, Washington D.C.

O'Reilly, W. (1984): Rock and Mineral Magnetism; Blackie, $230 \mathrm{pp}$.

Pucher, R. \& Rolf, C. (2001): Die Magnetfeldanomalie bei Messel: Interpretionsversuche. - 61. Annual Assembly Deutsch. Geophys. Ges., Abstract: 111; Frankfurt/M.

Richter, M. (1942): Geologie des Rodderberges südlich von Bonn. - Decheniana, 101 AB: 1-24; Bonn.

Reynolds, J. M. (1998): An introduction to applied and environmental geophysics: 127; Chichester (Wiley).

Roeser, H. A. (1963): Gesteinsmagnetische Untersuchungen an Blitzeinschlagstellen im Basalt. - Diplomarbeit Universität Göttingen.

VogELSAnG, D. (1958): Geomagnetisches Strukturbild des Rodderberg-Vulkans südlich Bonn. - N. Jb. Geol. Paläont. Mh 1: 21-25, 2 Abb.; Stuttgart.

SENGPIEL, K. P. (1981): Forschungsvorhaben "Ausrïstung und Erprobung eines Hubschraubers für geophysikalische Messungen " - Bericht BGR, Archiv Nr. 87 893; Hannover.

Thicmer, M. \& Trzkan, B. (2002): Geophysikalische Untersuchungen am Rodderberg-Vulkan bei Bonn. - 62. Jahrestagung Deutsch. Geophys. Gesell., Abstract: 75-76; Hannover.

Van den Bogaard, P., Schmincke, H. U., Hall, C. M. \& York, D. (1989): Homogeneity versus heterogeneity in tephra crystal populations. - Bulletin New Mexico Bureau of Mines and Mineral Resource: 25 (abstract), Gen. Assembl. Assoc. Volcanol. Chemistry Earth's Interior; Santa Fe, USA.
Zijperveid, J. D. A. (1967): A. C. demagnetization of rocks: analysis of results. In: COLinson, D. W., Creer, K. M. \& Runcorn, S.K. (Editors), Methods in Palaeomagnetism: 254-286; Newcastle. 